\title{
Development of Energy Efficient CoAP Based WSN Time Synchronization System Using NS2
}

\author{
Sukanya Padave ${ }^{1}$,Prof. Gayatri S. Ambadkar ${ }^{2}$, \\ ${ }^{I}$ (Student NMIET, Talegaon, Pune, Maharashtra, India) \\ ${ }_{2}^{2}$ (Assistant Professor, E\&TC, NMIET, Talegaon, Pune, Maharashtra, India)
}

\begin{abstract}
This paper aims to develop energy efficient solution for transmission in WSN. Sensors works on battery powered cells which is difficult to replace again and again due to excessive use of power while being active all the time. For time synchronization in the transmission of the data from several nodes to its intended destination COAP architecture is developed using NS2 along with that, developed architecture has been evaluated in the form of energy efficient model called as EECoAP protocol.
\end{abstract}

Keywords: Time Synchronization, WSN, CoAP (Constrained Application Protocol), EECoAP (Energy Efficient CoAP).

\section{Introduction}

Wireless technology is developing to higher level, as use of wireless channel to communicate over long distance is increasing day by day which is making it one of the most excellent and desirable development in communication technology. Being heart of any control or monitoring method, wireless sensor plays a vital role in transferring sensed data over the distance more quickly. Main task of the sensor node is depends on its configured threshold value to sense particular data where it has been installed to observe operation of application field. Monitoring any application keeps sensors active all the time which will expend more power than the capacity it has in its battery powered cells. Motivation behind developing algorithm is to save power i.e energy expenditure in WSN, we can call this algorithm as EECoAP(Energy Efficient Constrained Application Protocol) as mechanism to reduce the energy expenditure is developed for CoAP protocol, so the developed algorithm is labeled as Energy Efficient CoAP. Fig.1 is the explanation how nodes sends data to destination decision making device through home gateway.

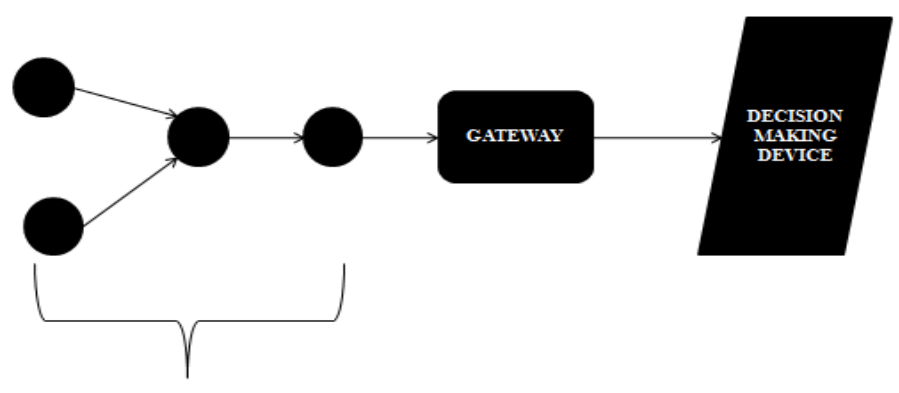

WIRELESS SENSOR NODES

Fig.1. Connection of wireless sensors to send data.

\section{Related Study}

Many researchers were working to find solution for reducing energy expenditure in WSN. As data transferring through internet that is with the help of IP and Non-IP devices energy saving is the prime important characteristic in today's development. Energy expenditure using Position Responsive routing Protocol with the help of GPS system can be reduce to much higher level[1]. Central node in WSN has capability to keep status of the node with the help of which path can be selected to reduce energy consumption in transferring the data[2]. Various energy resourceful methods like LEACH, HEED, DECA etc are discussed by[3]. How clustering method of WSN can help to reduce energy expenditure is explained in[4][10]. Maxwell equation can increase sensors life with the help of efficient routing protocol[2]. Network lifespan has been increased to good level by using rigorous approach in routing[5]. Fig.2 explains important sections in sensor node it comprises of sensing device which sends value sensed or recorded with the help of transducers i.e it accepts physical input signal and sends electrical output to processor and controller unit which is responsible to take appropriate action with the help of trans-receiver section all these major components works on non rechargeable batteries. 


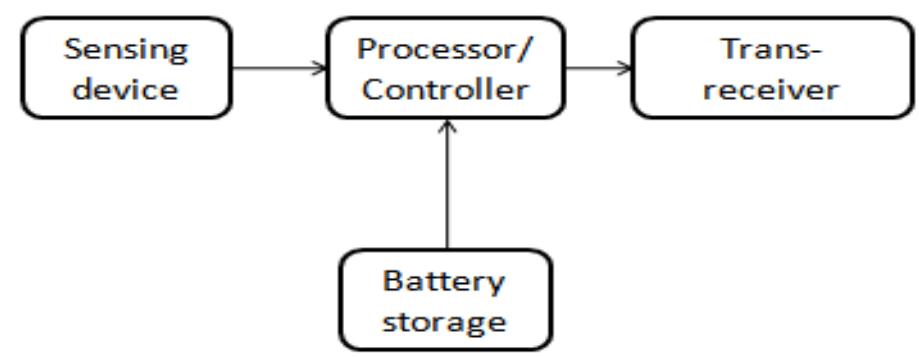

Fig.2.Wireless Sensor block diagram

\section{EECoAP System Model}

In this section we will discuss the work flow of designed Energy efficient method. Fig.3 explains that initially home automation environment is designed using NS2 i.e few nodes as sensor devices and gateway to communicate with end entity. Next important step is to get connected with other devices and that step is labeled as neighbor discovery. Third step is for synchronizing time with central system, in which initially time statistics is shared and depend on difference value compensation is done for accurate time matching. Piggybacking will help to utilize channel capacity. In between delay calculation and compensation one major step takes place that is selecting proper path depending on its highest residual energy capability. Once the path is decides with the help of minimum energy expenses handoff can be followed that means nodes will connect to gate way to transfer the status of surrounding recorded by sensor to end devices and depend on recorded status end entity can send the signal to take certain action for devices installed in application field. This all system will work together by coordinating each other only if based system who is responsible for time synchronization is working properly and for this purpose by considering efficiency of CoAP for managing time accuracy, CoAP protocol is the base for this method[6]. We are implementing this system on NS2 because there are many Network simulators available likeNS-2,NS-3,QualNet,MATLAb/simulink etc but after considering various performance parameters NS-2,NS-3 and QualNet are the best simulators that can be use in research work of WSN(Wireless Sensor Networks)[7].

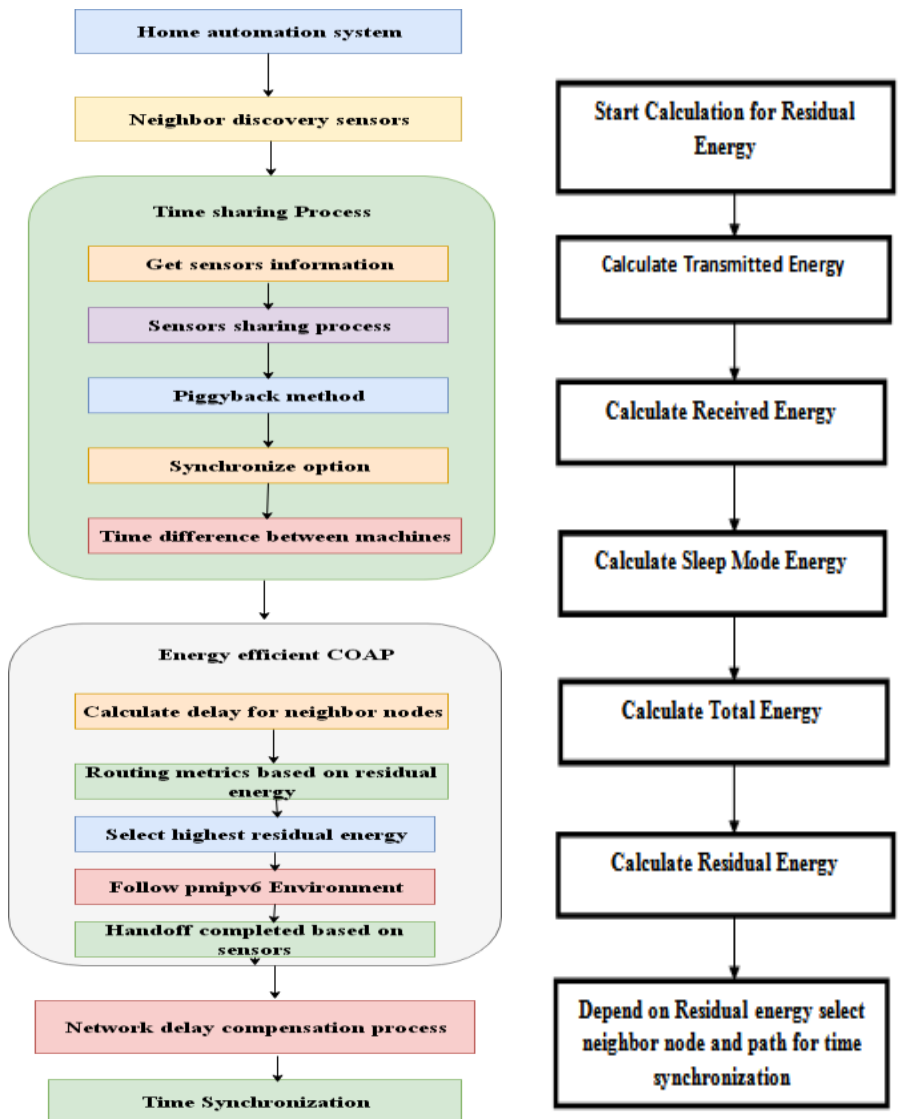

Fig.3. workflow of EECoAP protocol Fig.4.Algorithm for Energy calculation in EECoAP 


\section{System Implimentation And Evaluation}

In this section we will discuss how system For EECoAP is developed using NS2, However to compare the system along with EECoAP, basic CoAP[6]system and NTP[8] is also designed using NS2. First we will discuss basic algorithm designed for energy expenditure calculation and then system implementation for NTP, CoAP, and EECoAP respectively.Above Fig.4 gives the explanation of algorithm for Residual energy calculation. In which Transmitted, Received and the energy used while being in sleep mode is calculated. Addition of all these three energy is nothing but the total energy. With the help of total energy consumed and initial energy, residual energy is calculated.

Total Energy $=$ Transmitted energy + Received Energy + Sleep Energy

Residual Energy = Initial Energy - Total Energy

Once the Residual energy is calculated depend on that neighbor node and path to destination for time synchronization is selected.

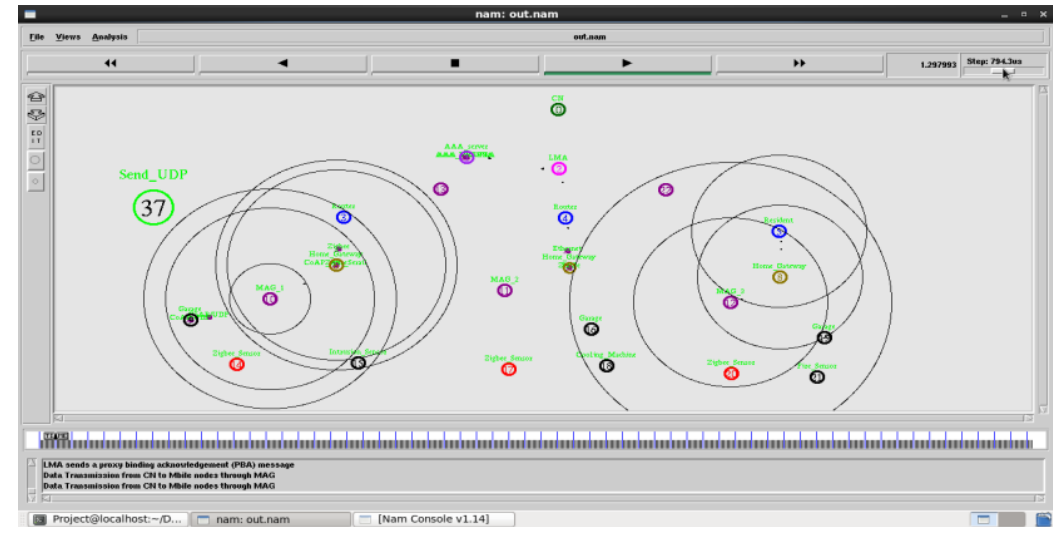

Fig.5. System Implementation For NTP, CoAP, EECoAP based System using NS2

Fig.5. is the experimental set up for NTP, CoAP, And EECoAP systems designed using NS2 software. Major difference between these three systems is NTP system is designed in which request response uses TCP based instructions which works on TCP/IP model, use of HTTP in NTP system increases its complexity which reduces its performance and that is the reason behind using another less complex protocol i.e CoAP. Advantage of being less complex and more efficient for CoAP protocol is because of UDP based request/ Response in transmission. Similar to CoAP , EECoAP also uses UDP based request response but the additional point in EECoAP is calculation of Residual energy and select path for transmission and synchronization depend on Residual energy. For designing Automation system we have added few nodes which can be installed in application field, for communication home gateway is added as another node which is responsible to transfer the information between sensor device and end entity. For example in this scenario in fig. 5 some threshold is set for node labeled as fire sensor when the condition met with high recorded temperature it generates alarm indicated by color changing of the sensor in this case as explained earlier info is transferred from node to residence through home gateway and vice versa.

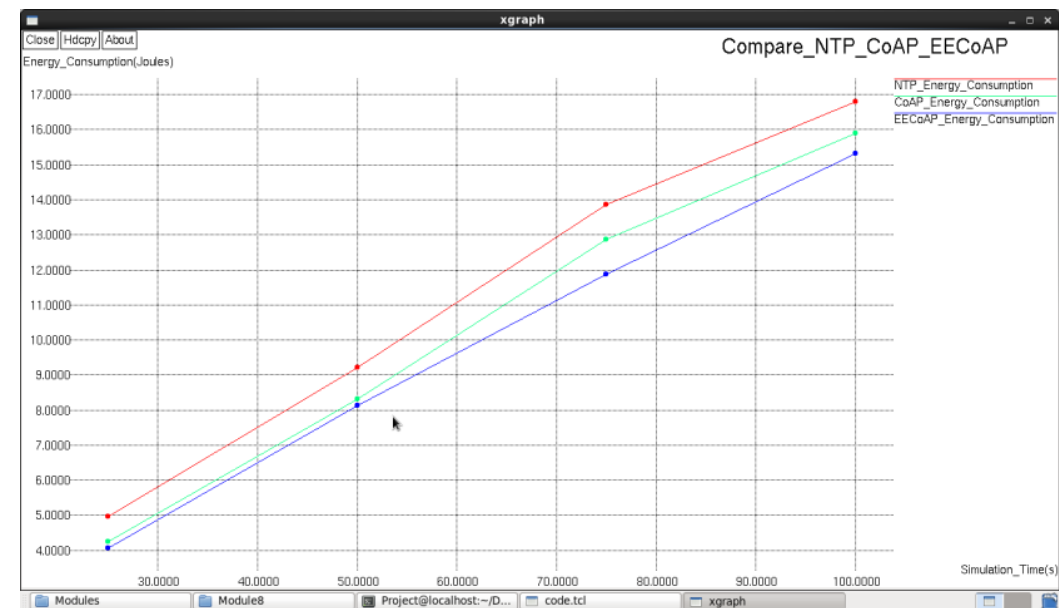

Fig.6.Comparision of Energy consumption in NTP, CoAP, EECoAP based systems 
Fig.6 is the explanation of energy consumption of all three systems in NS2.It has been observed that when residual Energy is considered to select the node and path for synchronization it reduces energy expenditure in EECoAP than NTP and CoAP based systems.

\section{Conclusion}

Reduced energy expenditure is possible in EECoAP because residual energy calculation helps to select path which reduces end to end delay, and increases packet delivery ratio and overall throughput of the system. Aim behind developing EECoAP is to reduce the energy expenditure in WSN, which has been observed that EECoAP energy utilization is much lesser than NTP and CoAP which can help to overcome the problem of limited battery life of sensor node.

\section{References}

[1]. Noor Zaman , Azween B Abdullah, "Energy Optimization through Position Responsive Routing Protocol (PRRP) in Wireless Sensor Network", International Journal of Information and Electronics Engineering, Vol. 2, No. 5, September 2012.

[2]. Mehdi Kalantari, Mark Shayman, "Energy Efficient Routing in Wireless Sensor Networks".

[3]. B.Baranidharan, B.Shanthi, "A Survey on Energy Efficient Protocols for Wireless Sensor Networks", International Journal of Computer Applications (0975 - 8887)Volume 11- No.10, December 2010.

[4]. Amjad K, Abu- Baker, "Energy -Efficient routing in cluster based wireless sensor Networks: Optimization and analysis", Jordan journal of Electrical Engineering volume 22016.

[5]. Shashidhar Rao Gandham, Milind Dawande, Ravi Prakash, S. Venkatesan, "Energy Efficient Schemes for Wireless Sensor Networks with Multiple Mobile Base Stations".

[6]. Seung-Chul Son, Nak-Woo Kim, Byung-Tak Lee, Chae Ho Cho, and Jo Woon Chong, "A Time Synchronization Technique for CoAP-based Home Automation Systems", IEEE Transactions on Consumer Electronics, Vol. 62, No. 1, February 2016.

[7]. Anand Nayyar, Rajeshwar Singh, "Comprehensive Review of Simulation Tools for Wireless Sensor Networks (WSNs)", Journal of Wireless Networking and Communications 2015, 5(1): 19-47 DOI: 10.5923/j.jwnc.20150501.03.

[8]. D. Mills, J. Martin, J. Burbank, and W. Kasch, "Network Time Protocol Ver. 4: Protocol and Algorithms Specification," IETF RFC 5905, 2010.

[9]. Brunda J S, Manjunath B S, Savitha B R, Ullas P, “Energy Aware Threshold based Efficient Clustering (EATEC) for Wireless Sensor Networks", International Journal of Computer Technology and Electronics Engineering (IJCTEE) Volume 2, Issue 4, August 2012. 